\title{
Papel da vitamina D no lúpus eritematoso sistêmico
}

\author{
The role of vitamin $D$ in systemic \\ lupus erythematosus
}

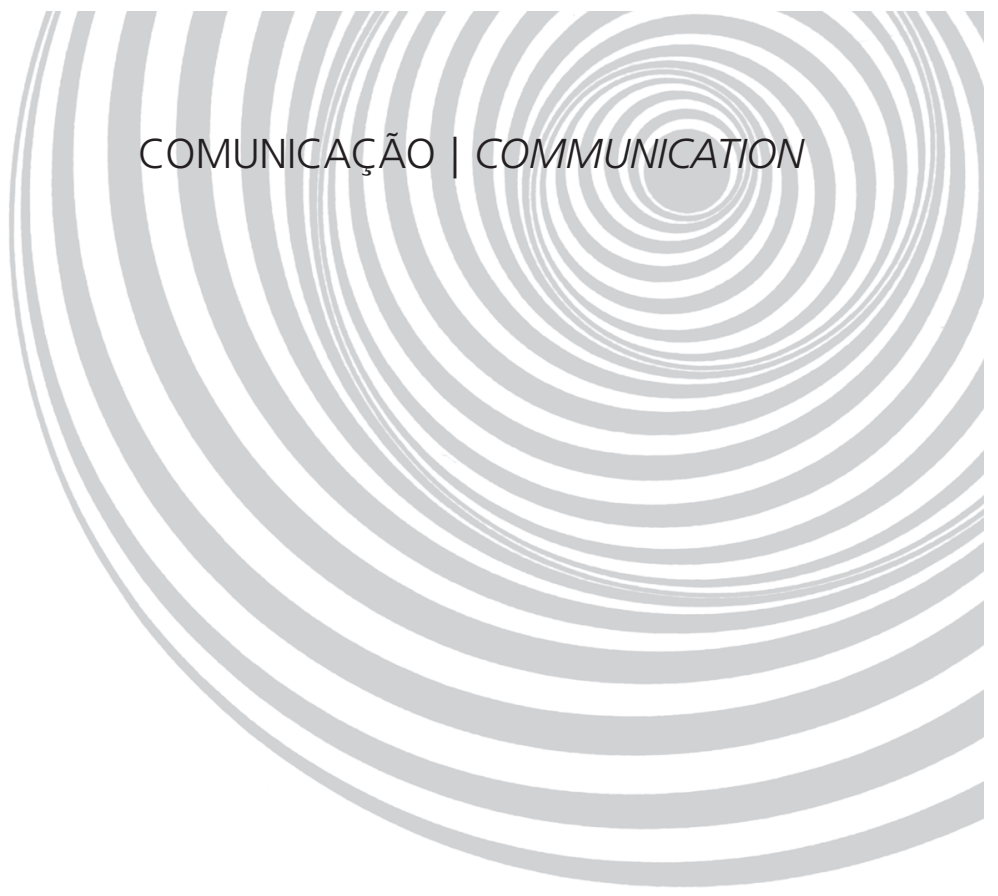

Thaisa de Mattos TEIXEIRA ${ }^{1}$

Célia Lopes da COSTA ${ }^{1}$

RE S U M O

Além do papel na homeostase do cálcio, acredita-se que a forma ativa da vitamina D apresente efeitos imunomoduladores, suprimindo ou ativando o sistema imune. Estudos recentes têm relacionado a deficiência ou a insuficiência de vitamina D a várias doenças autoimunes, como lúpus eritematoso sistêmico, artrite reumatoide, diabetes Mellitus tipo 1 e esclerose múltipla. Dessa forma, o presente trabalho buscou descrever o metabolismo da vitamina D e suas funções, enfatizando sua ação no sistema imune bem como a participação no lúpus eritematoso sistêmico. Todas as evidências encontradas estão voltadas para a relação entre baixos níveis de vitamina $D$ e manifestações clínicas do lúpus eritematoso sistêmico, porém ainda não está definido se a suplementação ou recuperação desses níveis possa influir na atividade da doença. Dessa forma, há necessidade de mais estudos para avaliar os possíveis benefícios terapêuticos da suplementação de vitamina $D$ no lúpus eritematoso sistêmico.

Termos de indexação: Lúpus eritematoso sistêmico. Sistema imune. Vitamina D.

\section{A B S T R A C T}

In addition to its role in calcium homeostasis, it is believed that the active form of vitamin $D$ has immunomodulatory effects, suppressing or activating the immune system. Recent studies have linked vitamin $D$ deficiency or insufficiency with multiple autoimmune diseases, including systemic lupus erythematosus, rheumatoid arthritis, type 1 diabetes Mellitus and multiple sclerosis. Thus, the present review describes vitamin $D$ metabolism and its functions, emphasizing its action on the immune system as well as its participation in systemic lupus erythematosus. All evidence focuses on the relationship between low vitamin D levels and the clinical manifestation of systemic lupus erythematosus, but it is not yet clear whether supplementation or reestablishment of normal levels can influence disease activity. Thus, more studies are needed to assess the possible therapeutic benefits of vitamin D supplementation on systemic lupus erythematosus.

Indexing terms: Immune system. Systemic lupus erythematosus. Vitamin D.

${ }^{1}$ Universidade do Estado do Rio de Janeiro, Instituto de Nutrição. R. São Francisco Xavier, 524, Pavilhão João Lyra Filho, $12^{\circ}$ andar, Bloco D, Sala 12 024, 20559-900, Rio de Janeiro, RJ, Brasil. Correspondência para/Correspondence to: CL COSTA. E-mail:<clcnut@terra.com.br>. 


\section{N T R O D U ÇÃ O}

A atuação da vitamina $D$ em processos metabólicos é pesquisada desde o século XVII, e foi objeto de prêmio Nobel em 1938. Atualmente, são conhecidos aproximadamente 41 metabólitos de vitamina $D$, sendo o 1,25-dihidroxicalciferol [1,25(OH)2D] um importante hormônio que atua como ligante para o fator de transcrição nuclear por meio do Receptor de Vitamina D (RVD), que regula a transcrição gênica e a função celular em diversos tecidos ${ }^{1}$.

Nos últimos anos, a vitamina $\mathrm{D}$ e seus pró-hormônios têm sido alvo de inúmeras pesquisas que comprovaram que sua função vai além do metabolismo do cálcio e da formação óssea. Essa ação sistêmica deve-se à expressão do RVD numa ampla variedade de tecidos corporais como cérebro, coração, pele, intestino, gônadas, próstata, mamas e células imunológicas, além de ossos, rins e paratireoides ${ }^{2}$.

A interação da vitamina D com o sistema imunológico parece se dar pela sua ação sobre a regulação e a diferenciação de células como linfócitos, macrófagos, células dendríticas, células T e B e células Natural Killer (NK), além de interferir na produção de citocinas in vivo e in vitro ${ }^{3}$.

São considerados quadros de deficiência níveis de 25-hidroxicalciferol [25(OH)D] abaixo de $20 \mathrm{ng} / \mathrm{ml}$, e de insuficiência, abaixo de $30 \mathrm{ng} / \mathrm{mL}$. Esses níveis são bastante comuns em pacientes com doenças crônicas autoimunes, incluindo Lúpus Eritematoso Sistêmico (LES), Artrite Reumatoide (AR), Diabetes Mellitus Tipo 1 (DMT1) e Esclerose Múltipla (EM)4,5.

O Lúpus Eritematoso Sistêmico é uma doença inflamatória crônica, de etiologia autoimune, multissistêmica, caracterizada por períodos de exacerbação e remissão ${ }^{6}$. Vários estudos comprovam que as concentrações séricas de vitamina $D$ estão associadas diretamente com a atividade da doença ${ }^{7}$. Dessa forma, tem sido sugerido que a vitamina $D$ possa ser um fator ambiental importante para a regulação do sistema imune, havendo necessidade da investigação do benefício da correção dessa deficiência. O objetivo da presente revisão bibliográfica, portanto, é descrever os mecanismos de ação da vitamina $D$ sobre o sistema imunológico, assim como estudar seus mecanismos de ação no LES.

A revisão foi realizada com base em artigos científicos de revistas indexadas. As publicações foram acessadas pelas bases de dados eletrônicas SciELO, Lilacs e MedLine, selecionadas nos idiomas português e inglês, utilizando-se os descritores: vitamina $D$, sistema imune, lúpus eritematoso sistêmico, doenças autoimunes, suplementação, 25-hidroxicalciferol, vitamin $D$, immune system, systemic lupus erythematosus, autoimmune disease, supplementation, 25-hydroxyvitamin D. Como critério de inclusão foram utilizados artigos publicados entre os anos de 1983 e 2012.

\section{Metabolismo e recomendações de vitamina D}

A vitamina $D_{3}$ ou colecalciferol é um hormônio esteroide encontrado em animais; sua função clássica consiste na manutenção do equilíbrio do metabolismo ósseo ${ }^{8}$. Essa vitamina também pode ser encontrada na forma vegetal como vitamina $D_{2}$ ou ergocalciferol e pode ser ingerida por meio da alimentação ou formada endogenamente em tecidos cutâneos após a exposição à radiação ultravioleta9.

A vitamina $D_{3}$ é produzida na pele pela irradiação ultravioleta do seu precursor, o 7-dehidrocolesterol. Durante essa exposição à radiação ultravioleta, o 7-dehidrocolesterol sofre conjugação de pontes de hidrogênio nos carbonos $\mathrm{C} 5 \mathrm{e}$ $C 7$, originando a pré-vitamina $D_{3}$. Essa molécula termolábil, após aproximadamente 24 horas, sofre rearranjo molecular dependente da temperatura, formando homodímeros, dando origem à vitamina $D_{3}$. A pré-vitamina $D_{3}$ também pode sofrer processo de isomerização, originando produtos biologicamente inativos (luminosterol e taquisterol), sendo esse mecanismo importante para evitar a superprodução de vitamina $D$ após períodos de prolongada exposição ao sol. O grau 
de pigmentação da pele é outro fator limitante para a produção de vitamina $D$, uma vez que peles negras apresentam limitação à penetração de raios ultravioletas. No sangue, o transporte da vitamina $D$ é feito principalmente através da proteína ligadora de vitamina $D$, e, em menor proporção, pela albumina ${ }^{10}$. No fígado, tanto a vitamina proveniente da dieta quanto a produzida endogenamente sofrem hidroxilação, mediadas pela enzima 25-hidroxilase, e são convertidas em 25(OH)D, que representa a forma circulante em maior quantidade, porém biologicamente iner$t^{10,11}$.

A $25(\mathrm{OH})$ D circula ligada à proteína ligadora da vitamina $D$ até o rim, onde ocorre a etapa final da produção do hormônio, e sofre uma nova hidroxilação, dando origem a 1,25(OH)2D, sua forma biologicamente ativa ${ }^{8,10}$.

Embora a forma ativa da vitamina $D$ seja a 1,25(OH)2D, a avaliação de níveis séricos é feita pela verificação da concentração da 25(OH)D, que está em maior quantidade e com meia-vida de aproximadamente três semanas. A 1,25(OH)2D normalmente não é dosada, uma vez que sua meia-vida é curta (aproximadamente $4 \mathrm{~h}$ ) e está em concentração mil vezes menor que a 25(OH)D. Além disso, no caso de deficiência de vitamina $D$, existe um aumento compensatório na secreção do Paratormônio (PTH), o que estimula o rim a produzir mais a 1,25(OH)2D. Desse modo, quando ocorre deficiência de vitamina $\mathrm{D}$ e queda dos níveis de 25(OH)D, as concentrações de 1,25(OH)2D se mantêm dentro dos níveis normais e, em alguns casos, até mesmo mais elevadas ${ }^{10,12}$.

Em 1989 e 1997, o Instituto de Medicina da Academia Nacional de Ciências ${ }^{13,14}$, fez recomendações sobre o consumo de vitamina $D$, porém estudos demonstraram uma ineficácia nas recomendações propostas ${ }^{15,16}$. Dessa forma, o Institute of Medicine (IOM) realizou uma revisão dos estudos relacionados à utilização de vitamina D e cálcio segundo as Referências de Ingestão Dietética (Dietary Reference Intakes - DRI) de 1997 e as evidências dos resultados para a saúde da população em diversos momentos fisiológicos.

Com isso, foram propostos novos valores de referência baseados em estudos de padrão-ouro, em que as recomendações foram baseadas em dose-resposta de eficácia para condições específicas de saúde (Tabela 1). No estudo supra-

Tabela 1. Referência Ingestão de Dietética para Cálcio e Vitamina D.

\begin{tabular}{|c|c|c|c|c|c|c|}
\hline Estágios da Vida & \multicolumn{3}{|c|}{ Cálcio } & \multicolumn{3}{|c|}{ Vitamina D } \\
\hline 0 a 6 meses & * & * & 1000 & ** & ** & 1000 \\
\hline 6 a 12 meses & * & * & 1500 & ** & ** & 1500 \\
\hline $4-8$ anos & 800 & 1000 & 2500 & 400 & 600 & 3000 \\
\hline $9-13$ anos & 1100 & 1300 & 3000 & 400 & 600 & 4000 \\
\hline $14-18$ anos & 1100 & 1300 & 3000 & 400 & 600 & 4000 \\
\hline $19-30$ anos & 800 & 1000 & 2500 & 400 & 600 & 4000 \\
\hline $31-50$ anos & 800 & 1000 & 2500 & 400 & 600 & 4000 \\
\hline 14 - 18 anos (gestantes/lactantes) & 1100 & 1300 & 3000 & 400 & 600 & 4000 \\
\hline 19 - 50 anos (gestantes e lactantes) & 800 & 1000 & 2500 & 400 & 600 & 4000 \\
\hline
\end{tabular}


citado, foi ressaltado que a evidência suporta a importância de vitamina D e do cálcio na promoção do crescimento e da saúde óssea, mas não em doenças como o câncer, doenças cardiovasculares, diabetes Mellitus tipo 2, obesidade e doenças autoimunes. No entanto, isso não significa que futuras pesquisas não revelem uma relação convincente entre vitamina $D$ e determinadas enfermidades ${ }^{17}$.

Além disso, novos dados indicam que o excesso desses nutrientes pode ser prejudicial. O comitê do IOM determinou que o risco de dano aumenta quando se consomem mais de $2000 \mathrm{mg}$ de cálcio ou mais que $4000 U$ I de vitamina D por dia. Dentre as complicações relacionadas com elevada ingestão, destaca-se a hipercalcemia ${ }^{18}$.

Tradicionalmente, níveis de 25(OH)D inferiores a $10 \mathrm{ng} / \mathrm{mL}$ ou $20 \mathrm{ng} / \mathrm{mL}$ são considerados pontos de corte para deficiência. Estudo recente definiu como deficiência níveis de 25(OH)D abaixo de $15 \mathrm{ng} / \mathrm{mL}$, de 15 a $30 \mathrm{ng} / \mathrm{mL}$ insuficiência e superior a $30 \mathrm{ng} / \mathrm{mL}$ como suficiente ${ }^{19-21}$.

A vitamina $D$ está pouco presente na alimentação da população mundial. As melhores fontes dietéticas de vitamina $D_{3}$ são peixes de água salgada (especialmente salmão, sardinha, arenque, atum e cavala), fígado e gema do ovo. Leite e manteiga são rotineiramente enriquecidos com vitamina $\mathrm{D}_{2}^{22}$.

\section{Ação da vitamina D sobre o sistema imunológico}

A identificação de RVD em várias células do sistema imune, entre elas linfócitos T, linfócitos $B$ e células dendríticas, despertou interesse pela vitamina $D$ como um fator imunorregulatório ${ }^{23,24}$. Nos linfócitos, as principais ações da vitamina D são: alteração na secreção de citocinas pró-inflamatórias, como a diminuição de Interferon-Gama (IFN- $\gamma$ ) e a Interleucina-2 (IL-2), bloqueando, assim, o principal sinal de retroalimentação das células dendríticas, gerando uma diminuição da capacidade de apresentação de antígenos aos linfócitos e diminuição da ativação e expansão clonal dos linfócitos. Ao mesmo tempo, ela aumenta a produção de IL-4, IL-5 e IL-10, e gera uma mudança de fenótipo T helper 1(Th1) para T helper 2(Th2), o que leva a uma maior tolerância imunológica ${ }^{25}$.

A ação da vitamina $D$ sobre o sistema imune também afeta a subpopulação de linfócitos Th17. Esses linfócitos T helper se caracterizam por secretar IL-17 e com isso participam na fisiopatogenia de doenças autoimunes. Outras citocinas também sofrem diminuição na sua produção na presença de vitamina $\mathrm{D}$, como IL-6, IL-12 e IL-2326.

As células Tregs reguladoras (células T) são críticas para a manutenção da tolerância imunológica e caracterizadas pela expressão elevada de $\mathrm{CD}^{+}$. Foi observado que a expressão das células T reguladoras está aumentada na presença de 1,25(OH)2D e que essas células se caracterizam por secretar IL-10 e prevenir o desenvolvimento de enfermidades autoimunes ${ }^{27}$.

Os linfócitos B também são alvo da ação da vitamina D: sobre essas células, eles têm efeitos diretos e potentes, estimulando a apoptose, inibindo sua proliferação e formação de células de memória, impedindo a diferenciação de células plasmáticas e síntese de imunoglobulinas ${ }^{26}$.

\section{Efeitos da vitamina $D$ sobre o lúpus eritematoso sistêmico}

O Lúpus Eritematoso Sistêmico é uma doença autoimune crônica, multissistêmica, caracterizada por acometimento de múltiplos órgãos e sistemas, cursando com períodos de exacerbação e remissão. Apesar da complexidade, a etiologia do LES ainda é pouco conhecida, sabe-se, porém, da importante participação de fatores genéticos, ambientais, hormonais e imunológicos para o surgimento da doença ${ }^{28,29}$. Embora sua distribuição seja universal, essa enfermidade é três vezes mais frequente em afro-americanos do que em brancos e dez vezes mais em mulheres ${ }^{7}$.

Vários autores têm demonstrado maior prevalência da deficiência de vitamina $D$ em 
pacientes com LES em comparação a indivíduos saudáveis ou com outras doenças reumatológicas ${ }^{7}$. Pacientes com LES apresentam múltiplos fatores de risco de deficiência de 25(OH)D. A fotossensibilidade característica da doença e a recomendação quanto ao uso de protetor solar determinam menor exposição do indivíduo ao sol, diminuindo a produção cutânea de vitamina $D$. $\mathrm{O}$ uso regular de corticosteroides e hidroxicloroquina parece alterar o metabolismo da vitamina $D$, embora as evidências ainda não sejam claras. Além disso, comprometimento renal grave que pode ocorrer nesses pacientes, com a presença de nefrite lúpica, pode alterar a etapa de hidroxilação da 25(OH)D ${ }^{30}$.

Pesquisas sugerem uma relação entre a deficiência da vitamina $D$ e o desenvolvimento da enfermidade. Ensaios in vitro mostraram que a suplementação de vitamina D diminui as anomalias características do LES ${ }^{31}$. Sugere-se que as alterações imunológicas causadas pelo deficit de vitamina $\mathrm{D}$ possam levar a uma diminuição da tolerância imunológica, permitindo o desenvolvimento de doença autoimune em indivíduos geneticamente predispostos.

A associação entre a deficiência de vitamina $D$ e a atividade de doença foi demonstrada por um estudo brasileiro com 36 pacientes com LES e 26 controles saudáveis. De acordo com os valores do índice de atividade da doença, Systemic Lupus Erythematosus Disease Activity Index (SLEDAI), os pacientes foram divididos em dois grupos: grupo 1, com SLEDAl médio de 22 (14-27), e grupo 2, com SLEDAl médio de 1,7 (0-3). A dosagem de 25(OH)D foi significativamente menor (Média - $M=17,4$, Desvio-Padão - DP=12,5ng/mL, $p<0,001)$ no grupo 1 quando comparada ao do grupo 2 ( $M=44,6, D P=14,5 \mathrm{ng} / \mathrm{mL}, p<0,001)$ ou com indivíduos saudáveis $(M=37,8, D P=13,7 \mathrm{ng} / \mathrm{mL}$, $p<0,001)$. Os pacientes com LES apresentaram uma alta prevalência de deficiência de $25(\mathrm{OH}) \mathrm{D}$, e isso foi negativamente relacionado com atividade de doença ${ }^{32}$.

Estudo transversal com 92 pacientes com LES evidenciou insuficiência de vitamina D
( $<30 \mathrm{ng} / \mathrm{mL}$ ) em $75 \%$ dos pacientes e deficiência $(<10 \mathrm{ng} / \mathrm{mL})$ em $45 \%{ }^{31}$. Esse estudo mostrou que deficiência e insuficiência de vitamina $D$ são comuns em pacientes com LES e que podem estar associadas à não exposição ao sol.

Em 2006, um estudo encontrou níveis críticos de vitamina $D$, definidos neste estudo como 25(OH)D abaixo de $10 \mathrm{ng} / \mathrm{mL}$, em 22 pacientes lúpicos dos 123 analisados, sendo os maiores preditores de risco a fotossensibilidade e a doença renal [Odds Ratio $(\mathrm{OR})=13,3, p<0,01 \mathrm{e}$ $\mathrm{OR}=12,9, p<0,01$, respectivamente]. Além disso, os níveis de $25(\mathrm{OH}) \mathrm{D}$ foram significativamente menores em afro-americanos em comparação aos caucasianos, mais altos no verão e menores no inverno ${ }^{33}$.

Com a investigação da presença de anticorpos antivitamina $\mathrm{D}$ no soro de 171 pacientes com LES para explicar melhor a deficiência de vitamina $D$ nas doenças autoimunes, foi detectada a presença do anticorpo em $4 \%$ dos pacientes com LES ${ }^{34}$. Apesar da baixa frequência de anticorpos antivitamina $D$ em pacientes com LES, os pesquisadores acreditam que esses anticorpos possam desempenhar um importante papel na deficiência de vitamina $D$, e que mais estudos são necessários para confirmar essa teoria em outras populações, utilizando-se esse anticorpo antivitamina $\mathrm{D}$ como marcador de diagnóstico e prognóstico.

Uma pesquisa avaliou a prevalência de deficiência ou insuficiência de vitamina $\mathrm{D}$ em pacientes coreanos portadores de LES. Além disso, foi analisada a relação entre os níveis de vitamina D com os marcadores da atividade da doença no LES e a influência do uso de corticoides na depleção de 25(OH)D. Os níveis séricos de 25(OH)D de pacientes com LES ( $M=42,49, D P=15,08 \mathrm{ng} / \mathrm{mL}$ ) foram significativamente menores do que os dos pacientes-controle $(M=52,72, D P=15,9 \mathrm{ng} / \mathrm{mL}$, $p<0,001)$. A frequência de insuficiência de vitamina $D$ foi significativamente alta em pacientes com LES quando comparada com a do controle normal $(p=0,032)$. Não houve diferenças significativas nas manifestações clínicas dos pacientes com LES com base nos níveis séricos de vitamina 
D. Além disso, não foram encontradas diferenças significativas nos níveis de vitamina $D$ entre os pacientes que faziam uso de corticoides e os que não faziam ${ }^{35}$.

Um estudo brasileiro avaliou a prevalência de insuficiência de 25(OH)D em 159 pacientes portadores de LES e analisou os fatores de riscos associados com a doença e a relação da insuficiência de vitamina $D$ e a atividade da doença. $A$ prevalência de insuficiência de vitamina $D$ foi encontrada em $37,7 \%$ dos pacientes (60 indivíduos), e de deficiência, em 8,2\% (13 pacientes). Os níveis de $25(\mathrm{OH}) \mathrm{D}$ tiveram uma correlação significativa com os níveis de PTH. O estudo não encontrou relação entre insuficiência de vitamina D e variáveis clínicas e demográficas como fotossensibilidade, uso de protetor solar, exposição ao sol e uso de suplementação oral de vitamina D. Além disso, o uso de corticoides e atividade da doença também não tiveram associação com a insuficiência de vitamina D. O estudo concluiu que, mesmo em um país tropical, uma alta prevalência de insuficiência de vitamina $D$ foi enconrada em pacientes com LES, e que intervenções terapêuticas não foram suficientes para prevenir insuficiência de vitamina $D^{36}$.

Em 2012, um estudo brasileiro ${ }^{37}$ avaliou a associação entre a insuficiência/deficiência de 25(OH)D com tempo de diagnóstico, fadiga, uso de corticosteroides, antimaláricos e antiDNA. Foram incluídos nesse estudo 78 pacientes portadores de LES e 64 controles saudáveis. Os níveis séricos médios de 25(OH)D foram 29,3ng/mL (6,1 - 55,2ng/mL) nos portadores de LES e 33,2ng/mL $(15,9-63,8 \mathrm{ng} / \mathrm{mL})$ nos controles saudáveis: essa diferença foi considerada estatisticamente significante $(p=0,041)$. Já em relação às outras variáveis avaliadas, não houve diferença estatisticamente significante (tempo de diagnóstico $p=0,343$; atividade da doença SLEDAI $p=0,971$; fadiga $p=0,808$; corticosteroides $p=0,701$; antimaláricos $p=0,986$; anti-DNA $p=0,435$ ).

A maioria dos estudos encontrados apenas correlaciona a deficiência de vitamina $\mathrm{D}$ e a atividade da doença. A suplementação de vitami- na D é necessária para a maioria, senão para todos os pacientes portadores de LES.

Um estudo fase I de suplementação oral de vitamina $D_{3}$ diária mostrou que doses de até 4 000Ul/dia durante 3 meses foram seguras e bem toleradas entre pacientes afro-americanos com LES. Os pesquisadores aguardam, contudo, os resultados de um estudo multicêntrico randomizado controlado de suplementação de vitamina $\mathrm{D}_{3}$ de $800 \mathrm{UI} / \mathrm{dia}, 2.000 \mathrm{UI} / \mathrm{dia}$ e $4.000 \mathrm{UI} / \mathrm{dia}$ para reduzir a expressão do IFN- $\alpha$ em pacientes com $\mathrm{LES}^{38}$.

\section{CONSIDERAÇÕ ES FINAIS}

Evidências sugerem que a vitamina $D$ tenha um grande potencial na regulação da resposta imunológica. Diversos estudos têm demonstrado que a deficiência e a insuficiência de vitamina $D$ estão presentes em várias doenças autoimunes, em especial no LES: seus níveis estão inversamente relacionados com a atividade de doença. Todas as evidências estão voltadas para a relação entre baixos níveis de vitamina $D$ e manifestação do LES, porém ainda não está definido se a suplementação ou recuperação desses níveis pode influir em maior período de remissão ou, ainda, pode ter uma repercussão na atividade da doença. Dessa forma, mais estudos são necessários para avaliar os possíveis benefícios terapêuticos da suplementação de vitamina D sobre as doenças autoimunes, em especial sobre o LES.

\section{COLABORADORES}

TM TEIXEIRA realizou a busca das referências bibliográficas em revistas indexadas, fez a redação do manuscrito, com posterior revisão criteriosa dos parágrafos e organizou as referências. CL COSTA participou como orientadora, realizou a revisão de trabalhos publicados nos últimos 20 anos, participou da redação e revisão do texto.

REFER Ê NCIAS

1. Bouillon R, Carmeliet $G$, Verlinden $L$, van Etten $E$, Verstuyf A, Luderer HF, et al. Vitamin D and human 
health: lessons from vitamin $D$ receptor null mice. Endocr Rev. 2008; 29(6):726-76.

2. Jones BJ, Twomey PJ. Issues with vitamin D in routine clinical practice. Rheumatol. 2008; 47(9): 1267-68.

3. Lemire JM, Ince A, Takashima M. 1,25-Dihydroxyvitamin D3 attenuates the expression of experimental murine lupus of MRL/1 mice. Autoimmunity. 1992; 12(2):143-8.

4. Holick MF. Vitamin D: importance in the prevention of cancers, type 1 diabetes, heart disease and osteoporosis. Am J Clin Nutr. 2004; 79(3):362-71.

5. Orbach H, Zandman-Goddard G, Amital H, Barak V, Szekanecz Z, Szucs G, et al. Novel biomarkers in autoimmune diseases: prolactin, ferritin, vitamin $D$, and TPA levels in autoimmune diseases. Ann N Y Acad Sci. 2007; 1109:385-400.

6. Lima I, Néri F, Santiago MB. Dosagem sérica de adenosina deaminase em lúpus eritematoso sistêmico: ausência de associação com atividade de doença. Rev Bras Reumatol. 2005; 45(5):273-9.

7. Kamen DL, Aranow C. The link between vitamin D deficiency and systemic lupus erythematosus. Curr Rheumatol Rep. 2008; 10(4):273-80.

8. Arnson $Y$, Amital $H$, Shoenfeld $Y$. Vitamin $D$ and autoimmunity: new etiological and therapeutic considerations. Ann Rheum Dis. 2007; 66(9): 1137-42.

9. Holick MF. Sunlight and vitamin D for bone health and prevention of autoimmune diseases, cancers, and cardiovascular disease. Am J Clin Nutr. 2004; 80(6 Suppl):1678S-88S.

10. Bringhurst FR, Demay MB, Kronenberg HM. Hormones and disorders of mineral metabolism. In: Kronenberg HM, Melmed S, Polonsky KS, Larsen PR, editors. Williams textbook of endocrinology. Philadelphia: Elsevier; 2008. v.11.

11. Leventis $P$, Patel $S$. Clinical aspects of vitamin $D$ in the management of rheumatoid arthritis. Rheumatol. 2008; 47(11):1617-21.

12. Bandeira F, Griz L, Dreyer P, Eufrazino C, Bandeira C, Freese E. Vitamin D deficiency: a global perspective. Arq Bras Endocrinol Metabol. 2006; 50(4):640-6.

13. National Academy of Sciences. Recommended Dietary Allowances [Internet]. Washington (DC): NAS; 1989 [cited 2010 Dec 15]. Avalilable from: <http://www.nap.edu/catalog.php?record_ $\mathrm{id}=1349>$.

14. Institute of Medicine. Dietary reference intake for calcium, phosphorus, magnesium, vitamin $\mathrm{d}$ and fluoride [Internet]. Washington (DC): IOM; 1997 [cited 2011 May 1]. Available from: <http://www. nap.edu/catalog.php?record_id=5776>.
15. Pittard WB, Geddes KM, Hulsey TC, Hollis BW. How much vitamin $D$ for neonates? Am J Dis Child. 1991; 145(10):1147-9.

16. Datta S, Alfaham M, Davies DP, Dunstan F, Woodhead S, Evans J, et al. Vitamin D deficiency in pregnant women from a non-European ethnic minority population: an international study. $\mathrm{Br} \mathrm{J}$ Obstet Gyneaecol. 2002; 109(8):905-8.

17. Institute of Medicine. Dietary reference intakes for calcium and vitamin D [Internet]. Washington (DC): IOM; 2010 [cited 2011 May 10]. Available from: <http://www.iom.edu/Reports/2010/DietaryReference-Intakes-for-Calcium-and-VitaminD.aspx>.

18. Vieth R. Vitamin D supplementation, 25hydroxyvitamin D concentrations, and safety. Am J Clin Nutr. 1999; 69(5):842-56.

19. Dawson-Hughes B, Heaney RP, Holick MF, Lips P, Meunier PJ, Vieth R. Estimates of optimal vitamin D status. Osteoporos Int. 2005; 16(7):713-6.

20. Chesney RW. Vitamin D and the magic mountain: the anti-infectious role of the vitamin. J Pediatr. 2010; 156(5):698-703 .

21. Holick MF. Vitamin D status: measurement, interpretation, and clinical application. Ann Epidemiol. 2009; 19(2):73-8.

22. Devlin TM. Princípios de nutrição Il: micronutrientes. In: Stephen GC, editor. Manual de bioquímica com correlações clínicas. São Paulo: Edgard Blücher Ltda; 2003. v.5.

23. Bhalla AK, Amento EP, Clemens TL, Holick MF, Krane SM. Specific high-affinity receptors for 1,25-dihydroxyvitamin D3 in human peripheral blood mononuclear cells: presence in monocytes and induction in $\mathrm{T}$ lymphocytes following activation. J Clin Endocrinol Metab. 1983; 57(6): 1308-10.

24. Provvedini DM, Tsoukas CD, Deftos LJ, Manolagas SC. 1,25-dihydroxyvitamin D3 receptors in human leukocytes. Science. 1983; 221(4616):1181-3.

25. Adorini L, Penna G. Control of autoimmune diseases by the vitamin $D$ endocrine system. Nat Clin Pract Rheumatol. 2008; 4(8):404-12.

26. Maskin $M$. La vitamina $D$, el sistema inmune y lãs enfermedades de la piel. Educ Med. 2009; 15(6): 401-09.

27. Szodoray P, Nakken B, Gaal J, Jonsson R, Szegedi $A$, Zold $E$, et al. The complex role of vitamin $D$ in autoimmune diseases. Scand J Immunol. 2008; 68(3):261-9.

28. D'Cruz DP, Khamashta MA, Hughes GR. Systemic Lupus Erythematosus. Lancet. 2007; 369(9561): 587-96. 
29. Pons-Estel GJ, Alarcon GS, Scofield L, Reinlib L, Cooper GS. Understanding the epidemiology and progression of systemic lupus erythematosus. Semin Arthritis Rheum. 2010; 39(4):257-68.

30. Cutolo M, Otsa K. Review: vitamin D, immunity and lupus. Lupus. 2008; 17(1):6-10.

31. Ruiz-Irastorza G, Egurbide MV, Olivares N, Martinez-Berriotxoa A, Aguirre C. Vitamin D deficiency in systemic lupus erythematosus: prevalence, predictors and clinical consequences. Rheumatol. 2008; 47(6):920-3;

32. Borba VZ, Vieira JG, Kasamatsu T, Radominski SC, Sato El, Lazaretti-Castro M. Vitamin D deficiency in patients with active systemic lupus erythematosus. Osteoporos Int. 2009; 20(3):427-33.

33. Kamen DL, Cooper GS, Bouali H, Shaftman SR, Hollis BW, Gilkeson GS. Vitamin D deficiency in systemic lupus erythematosus. Autoimmun Rev. 2006; 5(2):114-7.

34. Carvalho JF, Blank M, Kiss E, Tarr T, Amital H, Shoenfeld Y. Antivitamin D in SLE: preliminary results. Ann N Y Acad Sci. 2007; 1109:550-7.
35. Kim HA, Sung JM, Jeon JY, Yoon JM, Suh $\mathrm{CH}$. Vitamin D may not be a good marker of disease activity in Korean patients with systemic lupus erythematosus. Rheumatol Int. 2011; 31(9): 1189-94.

36. Souto M, Coelho A, Guo C, Mendonça L, Argolo $\mathrm{S}$, Papi J, et al. Vitamin D insufficiency in Brazilian patients with SLE: prevalence, associated factors, and relationship with activity. Lupus. 2011; 20(10): 1019-26.

37. Fragoso TS, Dantas AT, Marques CDL, Rocha Junior LF, Melo JHL, Costa AJG, et al. Níveis séricos de 25-HydroxivitaminaD3 e a sua associação com parâmetros clínicos e laboratoriais em pacientes com lúpus eritematoso sistêmico. Rev Bras Reumatol. 2012; 52(1)60-5.

38. Clinical Trials Studies. Vitamin D3 in systemic lupus erythematosus [Internet]. 2011 [cited 2011 May 10]. Available from: <http://clinicaltrials.gov/ct2/ show/NCT00710021>.

Recebido em: 3/2/2012

Versão final em: 10/5/2012

Aprovado em: 16/5/2012 